\section{The NCCN 2020 Virtual Congress: Hematologic Malignancies}

\author{
Robert W. Carlson, MD
}

$\mathbf{T}$ he treatment of hematologic malignancies has become increasingly complex as a result of the rapid pace and number of advances in the biology and treatment of hematologic malignancies. In addition, numerous new drugs have been approved or are currently in development, many with novel mechanisms of action. Clinicians who manage patients with hematologic malignances are challenged to stay abreast of advances in the evolving diagnostic and treatment landscape to ensure optimal patient outcomes.

Although the ongoing coronavirus pandemic continues to prevent in-person gatherings, the NCCN 2020 Virtual Congress: Hematologic Malignancies provided an opportunity for more than 850 hematologists, hematologist/oncologists, and other healthcare professionals to see world-renowned experts in the management of these malignancies present the latest updates in the NCCN Clinical Practice Guidelines in Oncology (NCCN Guidelines); new, emerging, and novel therapeutic agents; advances in cancer care; and the practical management of patients with hematologic malignancies. The virtual format for the congress provided opportunities for participants to engage with faculty in real time, and also included an online exhibit hall and breakout rooms for networking with colleagues. This special issue of JNCCN presents these Highlights as a synopsis of the proceedings.

The Virtual Congress, co-chaired by Ranjana H. Advani, MD, Stanford Cancer Institute, and Andrew D. Zelenetz, MD, PhD, Memorial Sloan Kettering Cancer Center, included important and timely topics, such as "Navigating the Jargon of Molecular Testing in Non-Hodgkin Lymphoma," "Challenges and Opportunities in Marginal Zone Lymphoma: Implications of Biology on Treatment," and "Relapsed/ Refractory Large B-Cell Lymphoma and Mantle Cell Lymphoma: Is It Time to Consider CAR-T for All?"

Additional sessions focused on controversies in the management of early-stage Hodgkin lymphoma, sequencing therapy and the role of minimal residual disease testing, emerging therapeutic options in acute lymphoblastic leukemia, evolving concepts in the diagnosis and staging of multiple myeloma, advances in acute myeloid leukemia management, and emerging therapeutic options in acute lymphoblastic leukemia, among others.

The presentations summarized herein present essential new information on how to achieve the best outcomes for patients, and also provide a deeper understanding of the strong evidence that supports recommendations found in the NCCN Guidelines. Our virtual platform allowed us to make vital cancer information more accessible than ever, as we adjusted for the ongoing COVID-19 pandemic. We value the fact that attendees from around the world and throughout the oncology ecosystem were able to join us from the convenience of their home or office without the need for travel.

This congress is just one of NCCN's educational events and programs that have continued despite the pandemic. For more information about educational opportunities, such as the upcoming NCCN 2021 Virtual Annual Conference, and NCCN programs such as the seminal NCCN Guidelines, NCCN Compendia, and NCCN Global endeavors, and to follow and participate in NCCN's continuing evolution, visit NCCN.org.

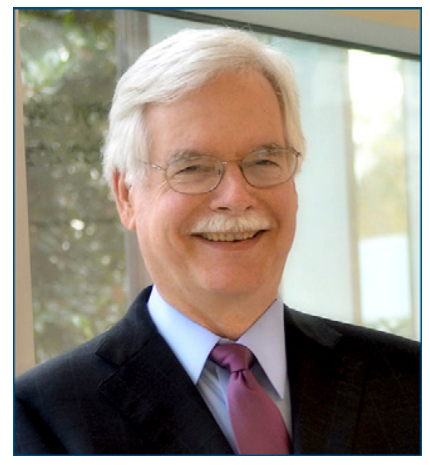

ROBERT W. CARLSON, MD

Robert W. Carlson, MD, joined NCCN as CEO in January 2013 following an esteemed history of leadership positions within the organization, including Representative to the NCCN Board of Directors, Chair of the Breast Cancer Guidelines Panel, and Member and Founding Chair of the Breast Cancer Risk Reduction Guidelines Panel. Prior to this appointment, he served as Professor of Medicine in the Division of Oncology and Stanford Medical Informatics at Stanford University Medical Center, as well as Medical Director of Inpatient Oncology and Hematology at Stanford Cancer Institute. Dr. Carlson is a graduate of Stanford University Medical School, and he completed his internship and junior residency in internal medicine at Barnes Hospital Group before returning to Stanford University to complete his senior residency. Dr. Carlson is board certified in Medical Oncology and Internal Medicine and is a member of several medical societies. He lectures extensively and has authored or co-authored more than 140 articles, abstracts, and book chapters. From 2001 through 2012, Dr. Carlson was selected by America's Top Doctors; from 1994 through 2011, he was selected by Best Doctors in America; in 2008, he was presented with the National Physician of the Year Award for Clinical Excellence by Castle Connolly Medical, Ltd.; and in 2010, Dr. Carlson was given the Founding Collaborator Award by the Breast Health Global Initiative (BHGI).

doi: $10.6004 /$ jnccn.2020.5030

The ideas and viewpoints expressed in this editorial are those of the author and do not necessarily represent any policy, position, or program of NCCN. 